\title{
Adverse Childhood Experiences among American Indian/Alaska Native Children: The 2011-2012 National Survey of Children's Health
}

\author{
Mary Kay Kenney and Gopal K. Singh \\ U.S. Department of Health and Human Services, Health Resources and Services Administration, Maternal and Child Health Bureau, \\ Rockville, MD 20857, USA
}

Correspondence should be addressed to Mary Kay Kenney; mkenney@hrsa.gov

Received 9 December 2015; Revised 25 May 2016; Accepted 31 May 2016

Academic Editor: Daniel Y. T. Fong

Copyright (C) 2016 M. K. Kenney and G. K. Singh. This is an open access article distributed under the Creative Commons Attribution License, which permits unrestricted use, distribution, and reproduction in any medium, provided the original work is properly cited.

\begin{abstract}
We examined parent-reported adverse childhood experiences (ACEs) and associated outcomes among American Indian and Alaska Native (AI/AN) children aged 0-17 years from the 2011-2012 National Survey of Children's Health. Bivariate and multivariable analyses of cross-sectional data on 1,453 AI/AN children and 61,381 non-Hispanic White (NHW) children assessed race-based differences in ACEs prevalence and differences in provider-diagnosed chronic emotional and developmental conditions, health characteristics, reported child behaviors, and health services received as a function of having multiple ACEs. AI/AN children were more likely to have experienced $2+$ ACEs (40.3\% versus $21 \%$ ), $3+$ ACEs (26.8\% versus $11.5 \%)$, $4+$ ACEs (16.8\% versus $6.2 \%)$, and $5+$ ACEs (9.9\% versus 3.3\%) compared to NHW children. Prevalence rates for depression, anxiety, and ADHD were higher among AI/AN children with 3+ ACEs (14.4\%, 7.7\%, and 12.5\%) compared to AI/ANs with fewer than 2 ACEs (0.4\%, $1.8 \%$, and 5.5\%). School problems, grade failures, and need for medication and counseling were 2-3 times higher among AI/ANs with 3+ ACEs versus the same comparison group. Adjusted odds ratio for emotional, developmental, and behavioral difficulties among AI/AN children with 2+ ACEs was 10.3 (95\% CI = 3.6-29.3). Race-based differences were largely accounted for by social and economic-related factors.
\end{abstract}

\section{Introduction}

A variety of deleterious child health and well-being outcomes have connections with adverse or traumatic experiences in childhood. Multiple experiences of food insufficiency and hunger are associated with behavioral, emotional, and academic problems and children exposed to family substance abuse and domestic violence show higher levels of aggression, delinquency, hyperactivity, impulsivity, anxiety, negative affectivity, and posttraumatic stress disorder compared to children without such histories [1-5]. Children exposed to maltreatment, family dysfunction, or caregiver loss frequently meet criteria for attention deficit hyperactivity disorder (ADHD) as well as conduct, anxiety, communication, and reactive attachment disorders [6]. A connection between levels of adversity in childhood and comorbid physical, mental, and substance abuse disorders in adulthood suggested that cumulative disadvantage is predictive of cumulative dysfunction $[7,8]$.

It has been stated that American Indians and Alaska Natives (AI/ANs) are disproportionally affected by childhood trauma, including abuse, neglect, and family violence, with pronounced disparities between White and AI/AN youth, sometimes attributed to cultural degradation resulting from multigenerational historical colonization and trauma [9]. One study of Native American adolescents and young adults from the Northern Plains states indicated that approximately half of the sample had been exposed to one or more severe traumatic events [10]. However, many AI/AN studies of outcomes for traumatized children have been conducted with nonrepresentative samples of adults or adolescents reporting on past experiences and examining narrowly defined health 
outcomes [10-13]. None have examined difficulties across a range of developmental, emotional, and behavioral problems experienced by younger children of varying ages.

The purpose of this study was to examine the prevalence of parent-reported adverse childhood experiences (ACEs) in a population-based nationally representative sample of AI/AN children from the 2011-2012 National Survey of Children's Health (NSCH). We examine ACEs in AI/AN children across the entire pediatric age spectrum of $0-17$ years and report on the associated emotional, developmental, and behavioral outcomes. Based on previous literature, it is hypothesized that (1) AI/AN race will be associated with greater accumulation of ACEs compared to a reference population of non-Hispanic White (NHW) children and (2) the increased accumulation of ACEs will be associated with an increasing gradient of parent-reported health problems and need for services among AI/AN children 0-17 years of age. Due to the high rates of mortality/morbidity among $\mathrm{AI} / \mathrm{ANs}$, including PTSD, suicide, and vehicular or violent injuries and death in adolescents and young adults, it is essential to find their roots in childhood to better prevent a self-perpetuating cycle of physical and behavioral health problems.

\section{Methods}

2.1. Population and Data. The NSCH is a quadrennial random-digit-dialing household survey that was designed to produce national and state-specific prevalence estimates of an array of variables concerning children and family (parental) health; children's physical, emotional, and behavioral development; family stress and coping behaviors; family activities; and parental concerns about their children. Of the original 95,677 cases in the survey, exclusions were made for cases with missing data for the adverse childhood experiences outcome measure, resulting in a sample of 94,520. The subpopulations of inference for the current analyses were 1,453 AI/AN 0-17-year-olds and a comparison group of 61,381 NHW children. The remaining sample was comprised of children of other races/ethnicities and was not included in this analysis. No oversampling of minority populations was conducted. Child-level household surveys were conducted with parents or guardians under the leadership of the Maternal and Child Health Bureau, Health Resources and Services Administration (HRSA), U.S. Department of Health and Human Services, and implemented through the National Center for Health Statistics, Centers for Disease Control and Prevention (CDC), U.S. Department of Health and Human Services.

All survey items/questions on the NSCH were developed under the direction of two technical expert working groups. The items were finalized after repeated rounds of cognitive testing as well as best practice language translation and pilot testing through the National Center for Health Statistics. All survey items used in this study have been documented previously and their properties are presented in publicly available manuals [14]. Data were weighted to represent the population of noninstitutionalized children aged 0-17 nationally and in each state. The National Center for Health
Statistics Research Ethics Review Board approved all data collection procedures for the survey.

2.2. Key Measures. The independent variables of interest included 9 different parent-reported ACEs calculated as individual rates and as accumulated rates from responses to the following survey questions: Has [child's name] ever (1) lived in a household with difficulty affording food or housing, (2) lived with a parent that had gotten divorced/separated, (3) lived with a parent who died, (4) lived with a parent who served time in jail, (5) seen parents hit, kick, slap, punch, or beat each other up, (6) been a victim of violence/witness to violence in [his/her] neighborhood, (7) lived with anyone who was mentally ill, suicidal, or severely depressed for more than a couple of weeks, (8) lived with anyone who had a problem with alcohol/drugs, and (9) been treated/judged unfairly based on race/ethnicity? The 9 NSCH adverse childhood experience survey items were based on items in the seminal work on adverse childhood experiences, with modifications overseen by a technical expert panel and evaluated through standard survey item testing through the National Center for Health Statistics [8]. Response alternatives were "yes/no" for most of the items with the exception of economic hardship and racial/ethnic discrimination experiences. Responses of "somewhat often" or "very often" (in contrast to "rarely" or "never") were coded as ever experiencing economic hardship and racial/ethnic discrimination.

Other key measures included the health, developmental, and service outcomes having possible associations with multiple ACEs. The survey questions and response alternatives on which these variables were based are listed in Table 1. These included (1) provider-diagnosed disorders (i.e., learning disability, depression, anxiety disorder, conduct disorder, autism spectrum disorder, attention deficit/hyperactivity disorder, developmental delay, and speech disorder) reported by the parent; (2) other parent-reported health characteristics (i.e., overall rate of special health care needs, prescription medication usage, elevated service use, functional limitations, special therapy usage, and emotional, developmental, or behavioral disorders requiring mental health treatment); (3) parent-reported behaviors in the $0-5$-year-old population (i.e., acquiring independence, learning in preschool, and behaving/getting along with others); (4) parent-reported behaviors in the 6-17-year-old population (i.e., school problems, frequent arguing, acting cruel/mean to others, unhappy, sad, or depressed affect, lack of control, investment in schoolwork, and grade repetition); and (5) health/services received (i.e., insured adequately, received needed counseling, screened by a doctor for developmental problems, screened for parent-reported developmental concerns, and received an Individualized Family Services Plan (IFSP) or Individualized Education Program (IEP)).

Finally, an additional outcome measure for logistic regression analysis was the proportion of parent-reported emotional, developmental, or behavioral difficulties. This was derived from one of the previously mentioned 5 questions used in a screening battery for children with special health care needs (i.e., Does [child's name] have any kind of emotional, developmental, or behavioral problem for which 


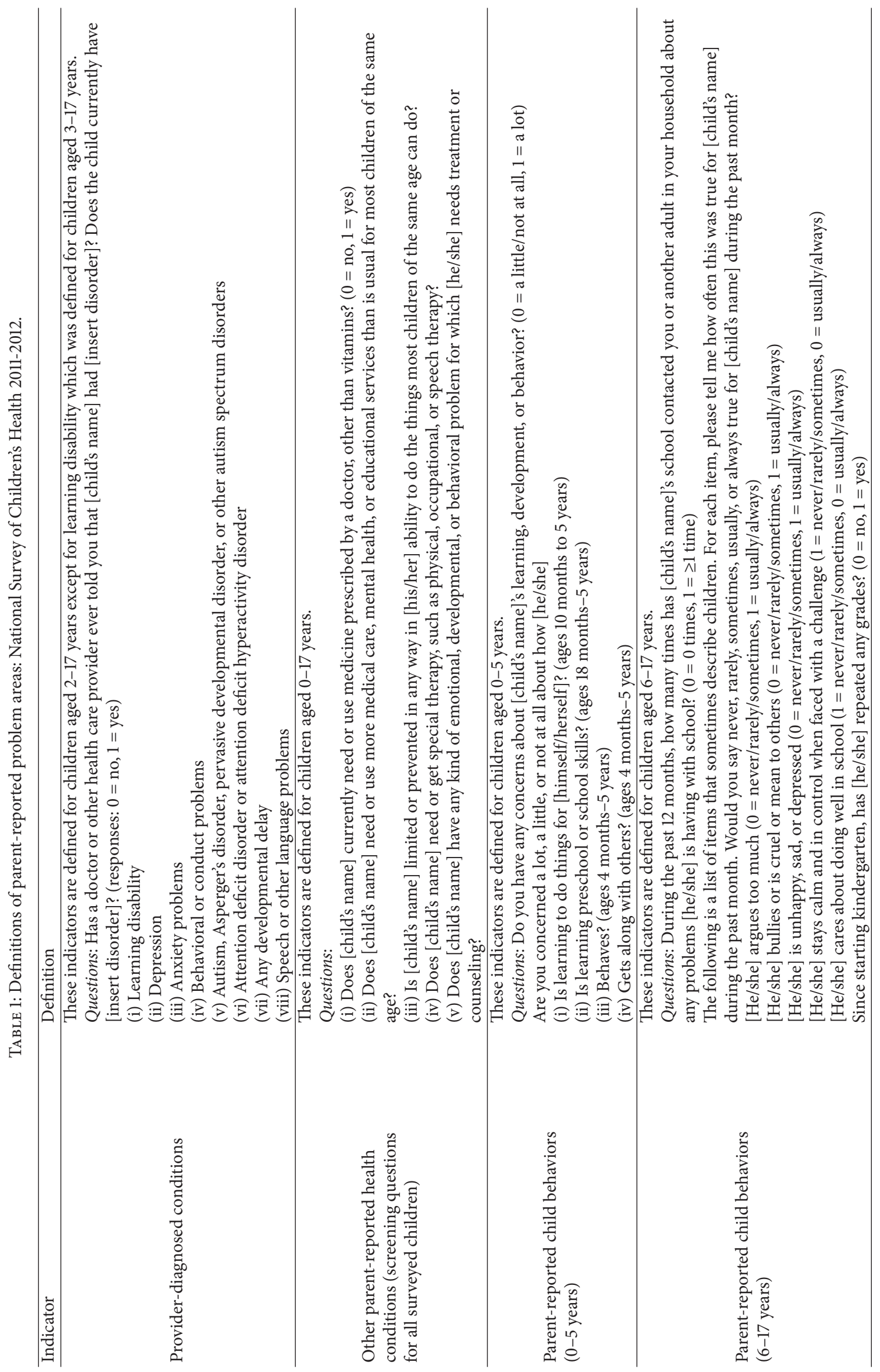




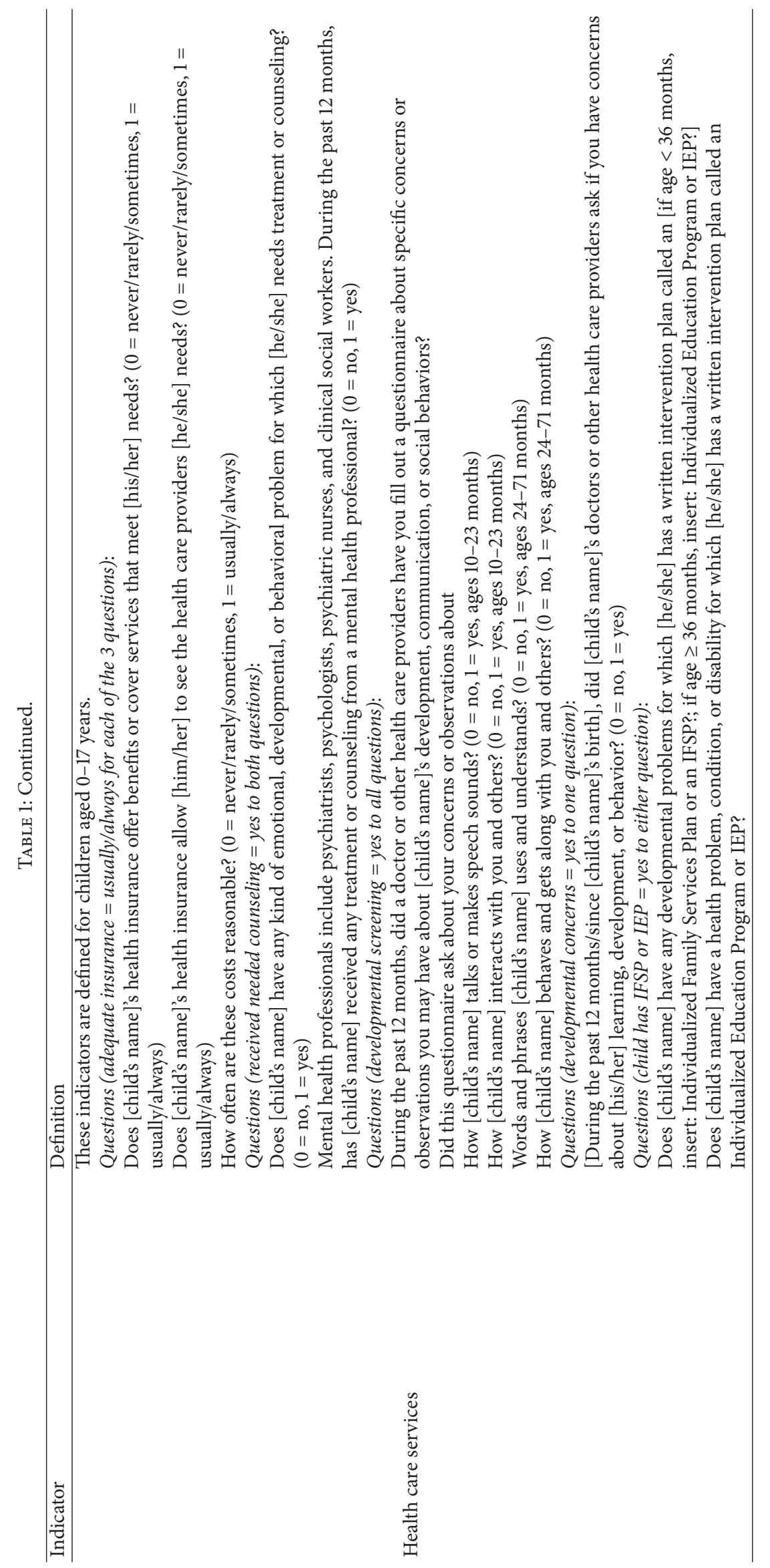


[he/she] needs treatment or counseling? Has [his/her] emotional, developmental, or behavioral problem lasted or is it expected to last 12 months or longer?). Response alternatives were "yes/no."

2.3. Sample Demographic Characteristics. Sociodemographic characteristics of the sample (Table 2) selected for race-group comparison included birth weight (very low or $<1500$ grams, low or 1500-2500 grams, and not low); gestational age (within normal limits, $\geq 3$ weeks premature); gender (male, female); age (0-5, 6-11, and 12-17 years); status of child with/without special health care needs which is a dichotomous variable based on a positive response to at least one of 5 survey items (i.e., child uses prescription medicine, needs special therapy, has elevated service use, has ongoing emotional, developmental, or behavioral conditions, or has limitations on activity); poverty status (<100\%, 100\%-199\%, 200\%$399 \%$, and $\geq 400 \%$ of the Federal Poverty level based on the U.S. Department of Health and Human Services Federal Poverty Guidelines for 2012); family structure (two parents, biological; two parents, step family; single mother, no father present; other); mother's age ( $\leq 30$ years, 31-45 years, or $>45$ years); highest household educational attainment ( $<$ high school, high school, and >high school); insurance coverage type (public, private, or none); medical home which is a dichotomous composite based on five component variables (i.e., having a personal doctor or nurse, having a usual source for sick and well care, having family-centered care, having no problems getting needed referrals, and receiving effective care coordination when needed) developed from 19 separate questions; neighborhood support, a dichotomous composite variable based on 4 survey items (i.e., people in the neighborhood help each other out, watch out for each other's children, can be counted on, and can be trusted to help a neighbor child who was outside playing and got hurt or scared); metropolitan status (metropolitan/nonmetropolitan residence); and AI/AN region (Alaska, East, Northern Plains, Pacific Coast, or Southwest). Weighted frequencies and prevalence were determined for the above characteristics for the AI/AN and NHW subpopulations.

2.4. Data Analysis Plan. We conducted the analyses of AI/AN ACEs and their relationships with a variety of demographic, health, developmental, and service factors in several steps. First, we determined differences between AI/AN children and a reference population (NHW children) in individual and accumulated, crude, and adjusted rates for the 9 parentreported ACEs (Table 3). The total number of ACEs (range: 0-9) was summed to create an overall crude ACE score per individual and then reported by the percentage of children with $0,1, \geq 2, \geq 3, \geq 4$, and $\geq 5$ ACEs. Relatively small numbers of $\mathrm{AI} / \mathrm{AN}$ necessitated using aggregate ACEs (e.g., $\geq 2, \geq 3$, $\geq 4$, and $\geq 5$ ) rather than single integrals of ACEs (e.g., 2, 3, 4 , and 5). $Z$ scores for the comparison of two proportions were used to determine the race-based differences in single and aggregate ACEs prevalence. Use of the NSCH ACE score has been established previously [15].

Second, we determined crude rate differences between AI/AN children with $<2$ ACEs compared to those with $\geq 2$ ACEs and $\geq 3$ ACEs as a function of selected health, developmental, and health service outcomes reported by the parent/caregiver (Table 4). The health, developmental, and service outcomes included those listed in Table 1. We calculated rate ratios and tested rate differences using the $Z$ test for the comparison of two proportions.

Finally, logistic regression was performed to assess factors associated with the cumulative ACEs within the AI/AN population while controlling for possible confounders (Table 5). Two different models were developed to explore associations between child, family, and environmental characteristics and the following outcomes: (1) 2 or more ACEs and (2) parentreported emotional, developmental, or behavioral conditions. The models produced adjusted odds ratios (AOR) for the sociodemographic characteristics (covariates) previously listed in Table 2. Multicollinearity diagnostics results did not identify problems with the inclusion of any of the above independent variables in the final models. The statistical analysis was conducted using SAS 9.3 survey procedures, which account for a complex sample design involving stratification, clustering, and multistage sampling.

\section{Results}

3.1. Sample. The sample characteristics are shown in Table 2 and indicate that, except for a limited number of variables (child's birth weight, gender, and CSHCN status), the AI/AN subsample was different in many ways compared to the NHW subsample. The AI/AN population had a higher percentage of children born $\geq 3$ weeks prematurely ( $15.7 \%$ versus $11.0 \%)$ and a lower percentage of $12-17$-year-olds (31.1\% versus $36.6 \%)$. At the family level, the AI/AN population had a $3-$ fold higher proportion of incomes below 100\% FPL (38.8\% versus $11.3 \%$ ) and a 3 -fold lower proportion of incomes $400+\%$ FPL (11.8\% versus $36.5 \%$ ). The AI/AN population was characterized by approximately 2.5 times higher proportion of households without a high school diploma (23.5\% versus $9.2 \%)$ and a higher percentage of mothers $<30$ years of age (39.3\% versus $22.9 \%)$. The household structure was more frequently reported to be a single mother with no father present $(22.6 \%$ versus $12.5 \%)$ or some other arrangement such as living with grandparents, other close relatives, or foster parents (15.0\% versus 5.9\%). The child's health care coverage was more frequently public ( $57.6 \%$ versus $23.5 \%$ ) or nonexistent (9.5\% versus $3.9 \%$ ) and the child was less likely to have a medical home (43.5\% versus $65.7 \%$ ). AI/AN families were more concentrated in nonmetropolitan statistical areas (40.3\% versus $20.1 \%$ ) and more likely to live in Alaska, the Northern Plains, and the Southwest compared to their NHW counterparts.

3.2. Prevalence of Adverse Childhood Experiences. Table 3 displays the individual and accumulated ACEs among AI/AN children and the comparison group of NHW children. Having been treated or judged unfairly based on race/ethnicity was approximately 7 times more common among AI/AN children than NHW children (10\% versus $1.4 \%)$. AI/AN children were 2-3 times more likely to have a parent who served time in jail (18\% versus 6\%), to have observed 


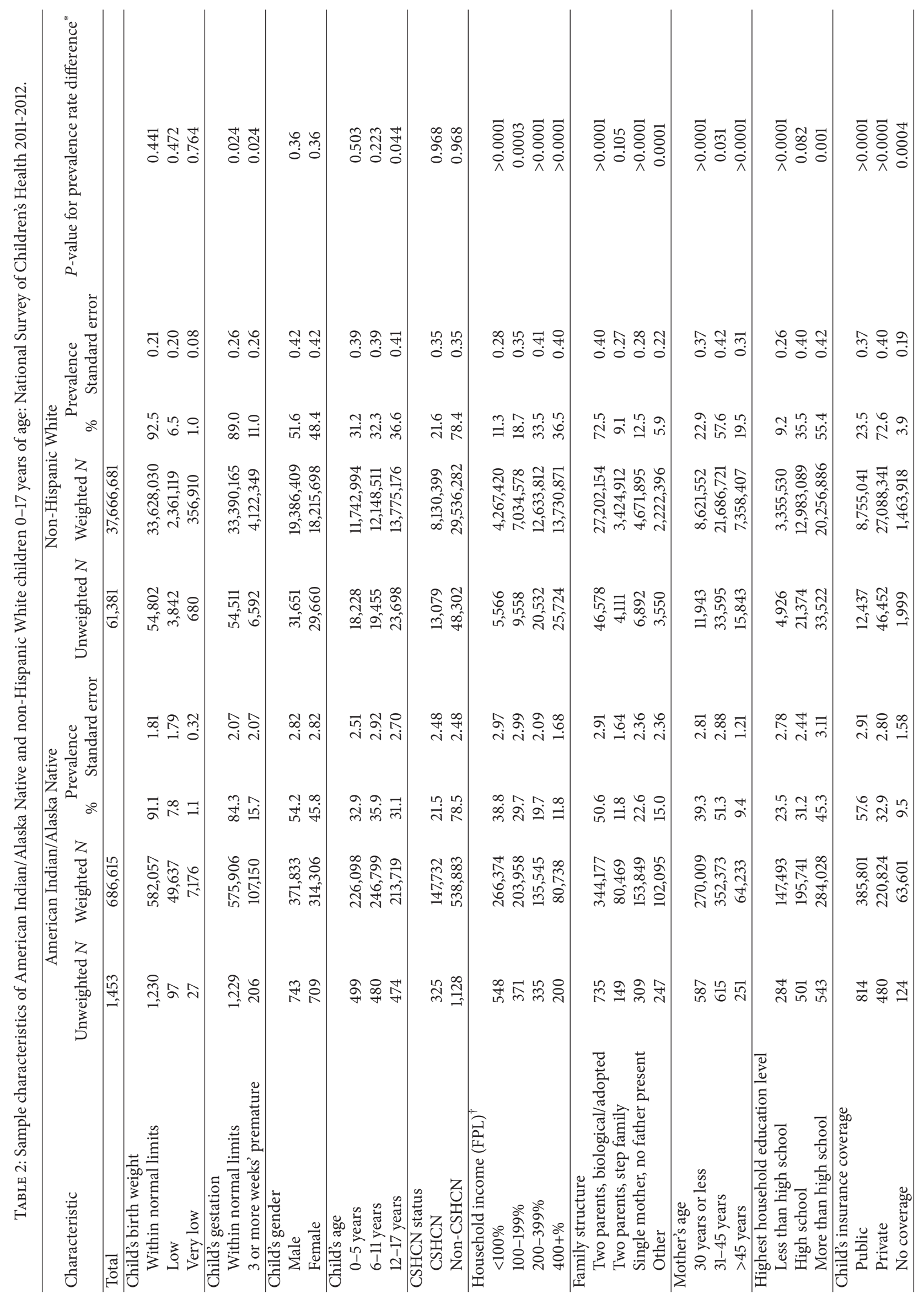




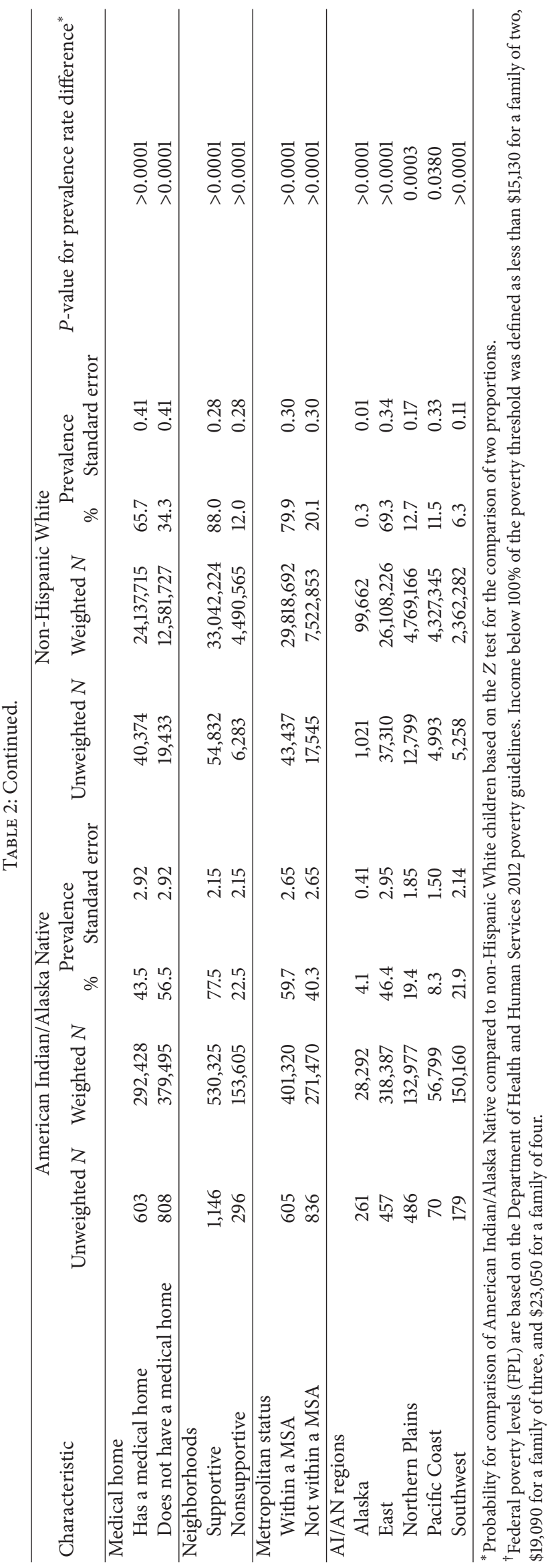




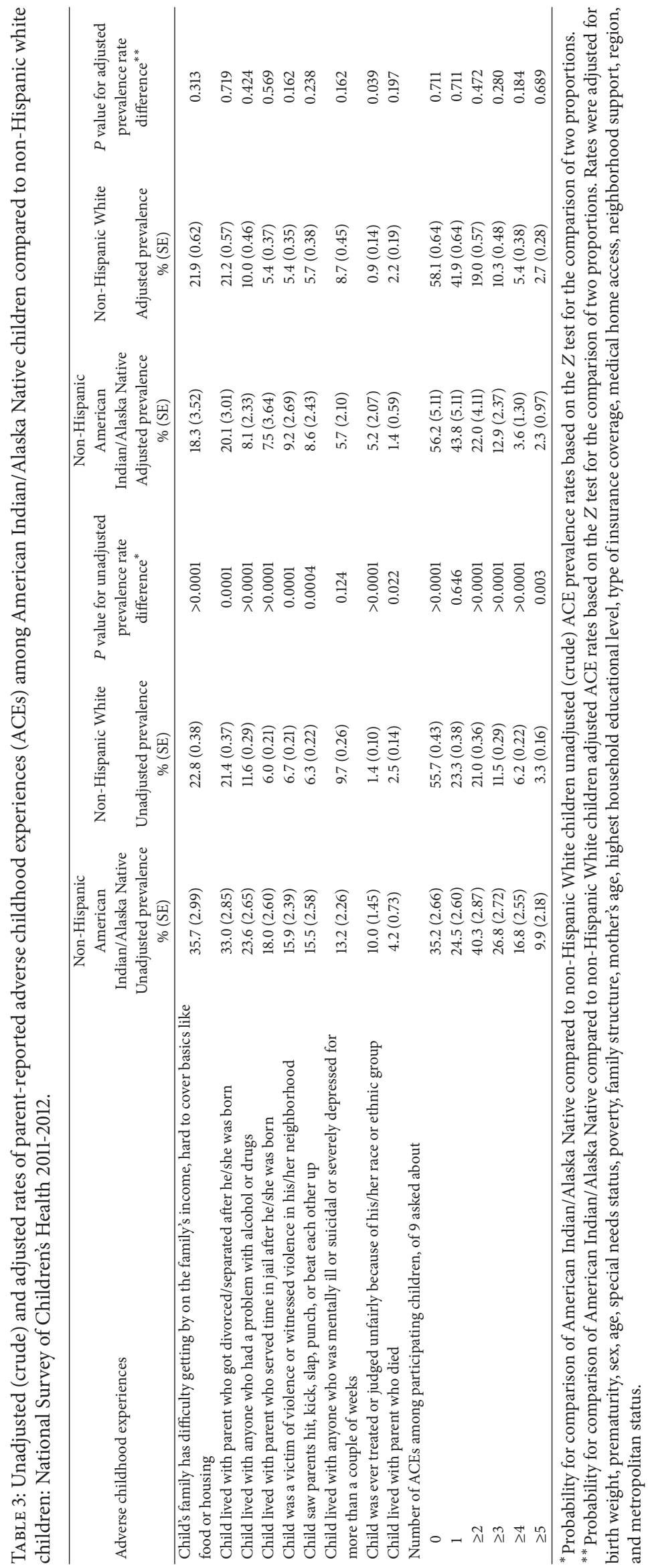


domestic violence ( $15.5 \%$ versus $6.3 \%$ ), to have been a victim of violence/witnessed violence in their neighborhood $(15.9 \%$ versus $6.7 \%)$, and to have lived with a substance abuser $(23.6 \%$ versus $11.6 \%)$. Finally, AI/AN children were 1.5 times more likely to live in families with difficulty covering basics like food or housing (35.7\% versus $22.8 \%$ ), to have lived with a divorced/separated parent (33\% versus $21.4 \%$ ), and to have lived with a parent who died (4.2\% versus $2.5 \%)$. AI/AN and NHW children were equally likely to have lived with a mentally ill/suicidal/severely depressed person for more than a couple of weeks (13.2\% versus 9.7\%). Differences in accumulated ACEs were also evident. AI/AN children were less likely to have none of the 9 adverse experiences queried (35.2\% versus $55.7 \%$ ) and equally likely to have had one experience (24.5\% versus $23.3 \%$ ) but $2-3$ times more likely to have multiple ( $\geq 2$ to $\geq 5$ ) ACEs (9.9 to $40.3 \%$ versus 3.3 to $21.0 \%$ ) compared to NHW children.

To control for the many sociodemographic differences (confounders) between the AI/AN and NHW children (Table 2), the prevalence rates for the individual and accumulated ACEs were adjusted for all sociodemographic variables. Adjusted rates are presented in Table 3 and indicate that all statistically significant differences between the two populations are eliminated after adjustment.

3.3. Health/Developmental Problems and Services among AI/AN Children with Multiple ACEs. Table 4 displays the prevalence rates, prevalence rate differences, and prevalence rate ratios (PRR) for various health, developmental, and service outcomes among AI/AN children with $<2$ ACEs, 2+ ACEs, and 3+ ACEs. Among the parent-reported providerdiagnosed conditions, depression and anxiety disorders were significantly more prevalent among AI/AN children with $2+$ ACEs (10.7\% and 6.3\%, resp.) compared with AI/AN children with $<2$ ACEs $(0.4 \%$ and $1.8 \%$, resp.) while these same emotional problems plus ADHD were more prevalent among AI/AN children with 3+ ACEs (14.4\%, 7.7\%, and $12.5 \%$, resp.) compared to AI/AN children experiencing fewer than 2 ACEs $(0.4 \%, 1.8 \%$, and 5.5\%, resp.). Prevalence rate ratios (PRR) for these same comparisons were the highest for depression (PRR = 26.8-36.0) and the lowest for ADHD (PRR $=2.0-2.3)$. AI/AN children with $<2$ ACEs were identified as having special health care needs 2.4 times less frequently than children with $2+$ ACEs (13.6\% versus 33.3\%) and 2.8 times less often than children with $3+$ ACEs (13.6\% versus $38.0 \%)$. The likelihood of using prescribed medications, having elevated service needs, and having functional limitations significantly increased by 2.2-3 times for children with $2+$ ACEs $(23.4 \%$, $14.4 \%$, and $5.7 \%$, resp.) and 2.1-3.6 times for children with $3+\operatorname{ACEs}(27.2 \%, 17.1 \%$, and $5.4 \%$, resp.) compared to those with $<2$ ACEs. In contrast, the likelihood of having a parentreported emotional, behavioral, and developmental problem was 11.2 times greater for children with 2+ ACEs (19.1\%) and 15.4 times for children with $3+$ ACEs (26.1\%) compared to children with $<2$ ACEs (1.7\%).

Differences in parent-reported behavioral concerns among 0-5-year-old AI/AN children with $<2$ ACEs were generally similar to the young children with $2+$ and $3+$ ACEs.
However, among AI/AN 6-17-year-olds, several parentreported behavioral concerns showed increases in children with $2+$ and $3+$ ACEs compared to those with <2 ACEs: having problems in school (PRR $=2.1$ and 2.0, resp.), arguing too much $(\mathrm{PRR}=2.7$ and 3.0, resp.), difficulty maintaining control in the face of challenges ( $P R R=2.7$ and 2.9, resp.), not caring about school performance $(\mathrm{PRR}=2.8$ and 2.8, resp.), and repeating grades ( $P R R=2.5$ and 2.9 , resp.).

Regarding health care and service needs, receiving needed treatment or counseling was associated with higher likelihood values with accumulated numbers of ACEs from less than 2 to $2+$ and $3+$ ACEs. Children with $2+(68.0 \%)$ and $3+(66.7 \%)$ ACEs were approximately 3.5 times more likely to have received needed counseling than children with less than 2 ACEs (19.6\%).

3.4. Associations with Multiple ACEs. The results of logistic regression to determine the relationship between individual, family, neighborhood, and residency factors and having $2+$ ACEs are shown in Table 5. Adjusted odds ratios (AOR) indicating positive associations included age $(\mathrm{AOR}=4.57$ for 6-11-year-olds and $\mathrm{AOR}=8.15$ for 12-17-year-olds), family structure $(\mathrm{AOR}=4.01$ for single mothers, no father present; $\mathrm{AOR}=4.85$ for other family structures), public insurance $(\mathrm{AOR}=2.23)$, neighborhood support $(\mathrm{AOR}=1.74$ for unsupportive neighborhoods), and region of the country $(\mathrm{AOR}=2.18$ for the Northern Plains states).

The results of an additional logistic regression analysis to determine the relationship between having emotional/developmental/behavioral problems as a function of individual, family, neighborhood, and residency factors and having 2+ ACEs are shown in Table 5. Results indicated that $\mathrm{AI} / \mathrm{AN}$ children with 2+ ACEs had approximately 10 times greater odds of having parent-reported emotional/developmental/behavioral problems than AI/AN children with $<2$ ACEs. Independent effects were also noted for birth weight $(\mathrm{AOR}=5.41$ for low/very low birth weight $)$ and family structure $(\mathrm{AOR}=0.34$ for single mother, no father present).

\section{Discussion}

The current analyses provide support, at least in part, for our hypotheses. First, we hypothesized that Native American children across the 0-17-year age range would be more likely to have greater accumulation of adverse experiences in childhood when compared to a reference population of NHW children in the same age range. Our results indicated that AI/AN children were more likely to have had 8 of 9 ACEs: income deprivation, witnessing or experiencing violent victimization, racial/ethnic discrimination, household substance abuse, domestic violence, parental incarceration, divorce, and death of a parent. Five of the 9 ACEs involved 2 - to 7-fold crude rate increases in likelihood compared to the NHW population. AI/AN children were more likely to have multiple ACEs $(\geq 2, \geq 3, \geq 4$, and $\geq 5)$ when compared to non-Hispanic White children. However, after adjusting for sociodemographic factors, rate differences were eliminated, 


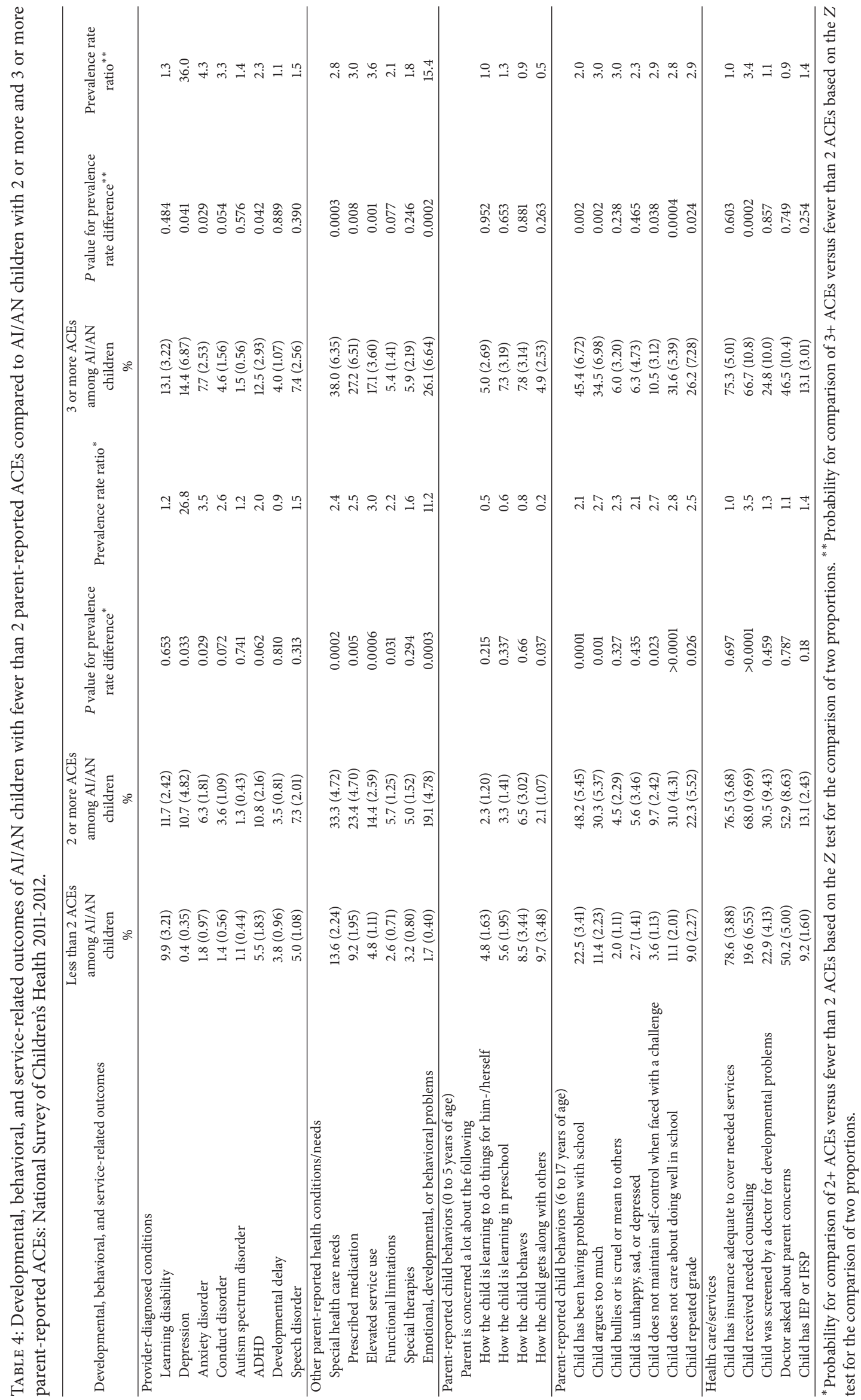


TABLE 5: Adjusted odds ratios for sociodemographic characteristics associated with (1) having 2 or more ACEs and (2) having parent-reported emotional/developmental/behavioral problems among AI/AN children 0-17 years of age: National Survey of Children's Health $2011-2012$.

\begin{tabular}{|c|c|c|c|c|}
\hline \multirow[t]{2}{*}{ Sociodemographic characteristics } & \multicolumn{2}{|c|}{$\begin{array}{l}\text { Outcome: two or more adverse family } \\
\text { experiences }\end{array}$} & \multicolumn{2}{|c|}{$\begin{array}{c}\text { Outcome: parent-reported } \\
\text { emotional/developmental/behavioral } \\
\text { problems }\end{array}$} \\
\hline & Adjusted odds ratio & $\begin{array}{l}\text { 95\% confidence } \\
\text { limits }\end{array}$ & Adjusted odds ratio & $95 \%$ confidence limits \\
\hline \multicolumn{5}{|l|}{ Adverse childhood events } \\
\hline 2 or more ACEs & na & na & 10.3 & $3.64-29.3$ \\
\hline Less than 2 ACEs & na & na & 1.00 & Reference \\
\hline \multicolumn{5}{|l|}{ Birth weight } \\
\hline Within normal limits & 1.00 & Reference & 1.00 & Reference \\
\hline Low/very low birth weight & 0.72 & $0.23-2.25$ & 5.41 & $1.48-19.8$ \\
\hline \multicolumn{5}{|l|}{ Child's gestation } \\
\hline Within normal limits & 1.56 & $0.60-4.05$ & 1.00 & Reference \\
\hline 3 or more weeks' premature & 1.00 & Reference & 1.61 & $0.48-5.37$ \\
\hline \multicolumn{5}{|l|}{ Child's gender } \\
\hline Male & 0.84 & $0.50-1.41$ & 1.30 & $0.60-2.81$ \\
\hline Female & 1.00 & Reference & 1.00 & Reference \\
\hline \multicolumn{5}{|l|}{ Child's age } \\
\hline $0-5$ years & 1.00 & Reference & 1.00 & Reference \\
\hline $6-11$ years & 4.57 & $2.23-9.36$ & 1.04 & $0.42-2.60$ \\
\hline $12-17$ years & 8.15 & $3.55-18.7$ & 1.89 & $0.66-5.43$ \\
\hline \multicolumn{5}{|l|}{${\text { Household income }(\mathrm{FPL})^{\dagger}}^{\dagger}$} \\
\hline$<100 \%$ & 1.51 & $0.51-4.47$ & 1.22 & $0.33-4.51$ \\
\hline $100-199 \%$ & 1.31 & $0.43-3.94$ & 1.71 & $0.49-5.94$ \\
\hline $200-399 \%$ & 1.09 & $0.42-2.80$ & 0.66 & $0.18-2.40$ \\
\hline $400+\%$ & 1.00 & Reference & 1.00 & Reference \\
\hline \multicolumn{5}{|l|}{ Family structure } \\
\hline Two parents, biological/adopted & 1.00 & Reference & 1.00 & Reference \\
\hline Two parents, step family & 2.04 & $0.97-4.32$ & 1.05 & $0.38-2.90$ \\
\hline Single mother, no father present & 4.01 & $2.00-8.03$ & 0.34 & $0.12-0.94$ \\
\hline Other & 4.85 & $1.99-11.8$ & 0.63 & $0.18-2.16$ \\
\hline \multicolumn{5}{|l|}{ Mother's age } \\
\hline 30 years or less & 1.06 & $0.41-2.70$ & 0.86 & $0.21-3.53$ \\
\hline $31-45$ years & 1.27 & $0.63-2.56$ & 1.30 & $0.46-3.65$ \\
\hline$>45$ years & 1.00 & Reference & 1.00 & Reference \\
\hline \multicolumn{5}{|l|}{ Highest household education level } \\
\hline Less than high school & 0.73 & $0.37-1.47$ & 0.88 & $0.38-2.90$ \\
\hline High school & 1.03 & $0.61-1.78$ & 0.88 & $0.39-1.96$ \\
\hline More than high school & 1.00 & Reference & 1.00 & Reference \\
\hline \multicolumn{5}{|l|}{ Child's insurance coverage } \\
\hline Public & 2.23 & $1.17-4.26$ & 1.13 & $0.43-2.95$ \\
\hline No coverage & 1.89 & $0.64-5.59$ & 0.91 & $0.21-3.95$ \\
\hline Private & 1.00 & Reference & 1.00 & Reference \\
\hline \multicolumn{5}{|l|}{ Medical home } \\
\hline Has a medical home & 1.00 & Reference & 1.00 & Reference \\
\hline Does not have a medical home & 1.17 & $0.70-1.94$ & 2.03 & $0.86-4.79$ \\
\hline
\end{tabular}


TABLE 5: Continued.

\begin{tabular}{|c|c|c|c|c|}
\hline \multirow[t]{2}{*}{ Sociodemographic characteristics } & \multicolumn{2}{|c|}{$\begin{array}{l}\text { Outcome: two or more adverse family } \\
\text { experiences }\end{array}$} & \multicolumn{2}{|c|}{$\begin{array}{l}\text { Outcome: parent-reported } \\
\text { emotional/developmental/behavioral } \\
\text { problems }\end{array}$} \\
\hline & Adjusted odds ratio & $\begin{array}{c}95 \% \text { confidence } \\
\text { limits } \\
\end{array}$ & Adjusted odds ratio & $95 \%$ confidence limits \\
\hline \multicolumn{5}{|l|}{ Neighborhoods } \\
\hline Supportive & 1.00 & Reference & 1.00 & Reference \\
\hline Nonsupportive & 1.74 & $1.01-3.00$ & 0.70 & $0.29-1.68$ \\
\hline \multicolumn{5}{|l|}{ Metropolitan status } \\
\hline Within a MSA & 1.00 & Reference & 1.00 & Reference \\
\hline Not within a MSA & 0.92 & $0.56-1.52$ & 0.58 & $0.26-1.32$ \\
\hline \multicolumn{5}{|l|}{$\mathrm{AI} / \mathrm{AN}$ regions } \\
\hline Alaska & 1.06 & $0.56-2.00$ & 1.69 & $0.68-4.21$ \\
\hline East & 1.00 & Reference & 1.00 & Reference \\
\hline Northern Plains & 2.18 & $1.21-3.94$ & 0.76 & $0.31-1.85$ \\
\hline Pacific Coast & 1.49 & $0.58-3.85$ & 1.95 & $0.51-7.53$ \\
\hline Southwest & 1.26 & $0.63-2.53$ & 0.69 & $0.24-1.98$ \\
\hline Wald $\chi^{2}$ statistic and $P$ value & 96.5 & $<0.0001$ & 46.6 & 0.005 \\
\hline$R^{2}$ (Nagelkerke max-rescaled $R$-square) & 0.85 & & 0.49 & \\
\hline
\end{tabular}

${ }^{\dagger}$ Federal poverty levels (FPL) are based on the Department of Health and Human Services 2012 poverty guidelines. Income below $100 \%$ of the poverty threshold was defined as less than $\$ 15,130$ for a family of two, $\$ 19,090$ for a family of three, and $\$ 23,050$ for a family of four.

suggesting that elevated risk among the AI/AN child population was substantially accounted for by a combination of child, family, neighborhood, and residency factors used to adjust the models.

The results of this investigation are difficult to compare to other studies due to methodological and procedural differences. A limited number of ACE studies have been conducted with the AI/AN population and those that have are often focused on nonrepresentative samples of mature adults or larger studies of adolescents reporting on their own, possibly distant, past experiences [10-13]. Furthermore, in most studies of $\mathrm{AI} / \mathrm{AN}$ adolescents and older $\mathrm{AI} / \mathrm{AN}$ populations, the presence of ACEs is related to long-term outcomes such as alcohol/drug addiction, suicide attempts, intimate partner violence, and incarceration in a previously ACE-exposed population, thus elucidating the cyclic nature of these experiences [10-12, 16-18]. In addition, these adverse experience studies typically reference a broader array of traumatic events than what is covered in the current survey (e.g., sexual and psychological abuse, as well as various forms of neglect and boarding school attendance).

Due to this and other methodological factors, the results reported here are somewhat at odds with findings of racebased differences in ACEs revealed by other studies with adolescents and older AI/AN populations. For example, heightened risk of multiple victimization was associated with Native American males in a nationally representative sample of adolescents [13]. In that study, AI/AN children 12 years of age or older in Indian Country experienced 2-3 times the victimization rate of Whites, Blacks, and Asians. Two-thirds of the victimization cases were across racial boundaries, indicating a considerable amount of racial discrimination.
Finally, rereferral rates to child protective services for abuse and neglect varied by racial and ethnic status with $\mathrm{AI} / \mathrm{AN}$ families having the highest rates [19]. While some of these studies may have controlled for a variety of factors, none included the same extensive list of sociodemographic variables included here. As indicated by the differences outlined in Tables 2 and 3, the social and economic disparities between the AI/AN and the NHW children may contribute heavily to the crude rate differences in adverse events observed for the two racial/ethnic subgroups of children.

Our second hypothesis stated that the increasing accumulation of adverse events among AI/AN children would be associated with a gradient of health problems and need for services in the AI/AN population. This hypothesis was supported to some extent by our findings that accompanying the higher accumulation of adverse experiences among the $\mathrm{AI} / \mathrm{AN}$ children was increasing prevalence of such problems reported by parents, particularly among children 6-17 years of age (e.g., arguing, lack of emotional control, and school problems) and more frequent provider-diagnosed behavioral disorders (depression, anxiety, and ADHD). AI/AN children with $2+$ and $3+$ ACEs received more medication and services such as counseling than AI/AN children with $<2$ ACEs.

The associated health and behavioral outcomes described here are reminiscent of those described by investigators of adolescents and older individuals. Similar to this investigation, one non-AI/AN study showed that, among younger (18-44 years), middle aged (45-64 years), and older (6589 years) adults, increased ACE scores were associated with increased prescription medication dispensing rates for the treatment of depression and anxiety [20]. A study of 7 $\mathrm{AI} / \mathrm{AN}$ tribes indicated a dose-response relationship with 
accumulated ACEs among AI/AN men and women [16]. That is, the number of different types of ACEs progressively increased the odds of having a negative outcome, such as alcohol dependence. Accumulated ACEs have also been found to be associated with increasing odds of attempted suicide and acts of violence among AI/AN women [12]. The diagnosis of PTSD (posttraumatic stress disorder) among $\mathrm{AI} / \mathrm{AN}$ adolescents is also related to the number of adverse experiences [11].

To overcome the trauma of ACE in AI/AN communities, it has been suggested that a continuum of prevention strategies addressing primary, secondary, and tertiary needs is strongly needed [11]. First, efforts must be made to prevent new ACE occurrences; secondly, where ACEs have already occurred, efforts must focus on the prevention of risky behaviors in response to those experiences. Finally, those who have already developed a health problem as a long-term consequence of ACEs will need help to change health risk behaviors in order to lower the potential for disease burden. Furthermore, prevention and treatment behavioral health efforts must be (1) addressed by individuals, families, and communities, (2) integrated into community health systems, and (3) founded on evidence-, culture-, and practice-based approaches [21].

A few recent approaches introduced into health and social systems in AI/AN communities reflect adherence to these principles. In some cases, this has been accomplished by adapting evidence-based programs to incorporate AI/AN cultural values. For example, the Indian Country Child Trauma Center developed AI/AN adaptation of the evidencebased treatment, trauma-focused cognitive-behavioral therapy. Honoring Children, Mending the Circle (HC-MC) guides the therapeutic process through blending of $\mathrm{AI} / \mathrm{AN}$ traditional teachings with cognitive-behavioral methods [22]. In other cases, nonadapted treatment protocols have been shown experimentally to be equally effective for an AI/AN subpopulation as for the targeted populations as a whole [23]. Finally, some programs have been specifically formulated for implementation in the AI/AN community.

Family Spirit intervention is an evidence-based AI/AN teen mother tribal home visiting program designed to address behavioral health disparities among American Indians and evaluated by using measures of intervention fidelity and early childhood emotional/behavioral development [24]. The latter targets maternal health, child development, school readiness, and positive parenting practices which are areas of emphasis in the Patient Protection and Affordable Care Act, Section 2951, addressing maternal, infant, and early childhood home visiting programs [25]. It is anticipated that home visiting programs in particular will address some of the underlying early life contributors to poor health and developmental outcomes for children, as well as persistent inequalities in the health and well-being of children and families. As the findings of this study indicate, the social determinants, especially those operating at the neighborhood, community, and regional levels, should figure prominently into the design of interventions aimed at improving the health of children and families. For example, the heavy concentration of unemployed AI/AN families relative to White families
(15\% versus $4.6 \%$ or 3.3 American Indian-to-White ratio) measured during the first half of 2013 in the Northern Plains region may have had some bearing on the doubling of the odds for ACEs compared to the East.

The findings presented here are subject to several limitations. The cross-sectional nature of the data imposes limits on the ability to discern any causal relationship between ACEs and the associated behaviors included here. In addition, due to the remote location and lower than average telephone service among many AI/AN families, sampling bias may reduce the representative nature of the AI/AN child sample from which estimates were drawn. Additional weaknesses include reliance on parent report for assigning children to diagnostic categories and evaluating functionality. These requirements may be difficult for parents in general but also may be fundamentally different for AI/AN parents compared to non-AI/AN parents based on cultural differences in the perception of disability that may lead to underreporting.

\section{Conclusion}

We have shown that significantly more AI/AN children 017 years of age are subject to adverse childhood experiences at a rate considerably higher and with greater complexity than a reference population of non-Hispanic White children. Increases in disease burden accompany those higher rates of adverse childhood experiences. Risks for some emotional, developmental, and behavioral problems in $\mathrm{AI} / \mathrm{AN}$ children were increased relative to the reference group, though these were determined to be accounted for by social and economic factors. AI/AN children are more likely to experience multiple adverse events as they develop and their health behaviors are being shaped. Greater attention to the social determinants of health at the family, neighborhood, community, and higher levels of contextual influences such as those operating at the state, regional, or national levels is needed to address the marked health disparities shown here.

\section{Disclosure}

The views in this paper are those of the authors and not necessarily those of the Health Resources and Services Administration of the U.S. Department of Health and Human Services.

\section{Competing Interests}

The authors have no financial interests relevant to this paper to disclose.

\section{References}

[1] R. E. Kleinman, J. M. Murphy, M. Little et al., "Hunger in children in the United States: potential behavioral and emotional correlates," Pediatrics, vol. 101, no. 1, article E3, 1998.

[2] M. M. Dore, E. Kauffman, L. Nelson-Zlupko, and E. Granfort, "Psychosocial functioning and treatment needs of latency-age children from drug-involved families," Families in Society, vol. 77, no. 10, pp. 595-603, 1996. 
[3] P. R. Giancola and A. M. Parker, "A six-year prospective study of pathways toward drug use in adolescent boys with and without a family history of a substance use disorder," Journal of Studies on Alcohol, vol. 62, no. 2, pp. 166-178, 2001.

[4] S. Maynard, "Growing up in an alcoholic family system: the effect on anxiety and differentiation of self," Journal of Substance Abuse, vol. 9, no. 1, pp. 161-170, 1997.

[5] S. A. Graham-Bermann and A. A. Levendosky, "Traumatic stress symptoms in children of battered women," Journal of Interpersonal Violence, vol. 13, no. 1, pp. 111-128, 1998.

[6] A. Cook, J. Spinazzola, J. Ford et al., "Complex trauma in children and adolescents," Psychiatric Annals, vol. 35, no. 5, pp. 390-398, 2005.

[7] J. P. Mersky, J. Topitzes, and A. J. Reynolds, "Impacts of adverse childhood experiences on health, mental health, and substance use in early adulthood: a cohort study of an urban, minority sample in the U.S.," Child Abuse \& Neglect, vol. 37, no. 11, pp. 917-925, 2013.

[8] V. J. Felitti, R. F. Anda, D. Nordenberg et al., "Relationship of childhood abuse and household dysfunction to many of the leading causes of death in adults. The adverse Childhood Experiences (ACE) Study," American Journal of Preventive Medicine, vol. 14, no. 4, pp. 245-258, 1998.

[9] T. W. Pavkov, L. Travis, K. A. Fox, C. B. King, and T. L. Cross, "Tribal youth victimization and delinquency: Analysis of Youth Risk Behavior Surveillance Survey Data," Cultural Diversity and Ethnic Minority Psychology, vol. 16, no. 2, pp. 123-134, 2010.

[10] A. J. Boyd-Ball, S. M. Manson, C. Noonan, and J. Beals, "Traumatic events and alcohol use disorders among American Indian adolescents and young adults," Journal of Traumatic Stress, vol. 19, no. 6, pp. 937-947, 2006.

[11] P. B. Deters, D. K. Novins, A. Fickenscher, and J. Beals, "Trauma and posttraumatic stress disorder symptomatology: patterns among American Indian adolescents in substance abuse treatment," American Journal of Orthopsychiatry, vol. 76, no. 3, pp. 335-345, 2006.

[12] L. De Ravello, J. Abeita, and P. Brown, "Breaking the cycle/mending the hoop: adverse childhood experiences among incarcerated American Indian/Alaska native women in New Mexico," Health Care for Women International, vol. 29, no. 3, pp. 300-315, 2008.

[13] T. N. Stevens, K. J. Ruggiero, D. G. Kilpatrick, H. S. Resnick, and B. E. Saunders, "Variables differentiating singly and multiply victimized youth: results from the national survey of adolescents and implications for secondary prevention," Child Maltreatment, vol. 10, no. 3, pp. 211-223, 2005.

[14] S. J. Blumberg, E. B. Foster, A. M. Frasier et al., "Design and operation of the National Survey of Children's Health, 2007," Vital and Health Statistics. Serious 1, Programs and Collection Procedures, vol. 55, pp. 1-149, 2012.

[15] K. S. Balistreri, "Adverse childhood experiences, the medical home, and child well-being," Maternal and Child Health Journal, vol. 19, no. 11, pp. 2492-2500, 2015.

[16] M. P. Koss, N. P. Yuan, D. Dightman et al., "Adverse childhood exposures and alcohol dependence among seven Native American tribes," American Journal of Preventive Medicine, vol. 25, no. 3, pp. 238-244, 2003.

[17] S. M. Manson, J. Beals, S. A. Klein, and C. D. Croy, "Social epidemiology of trauma among 2 American Indian reservation populations," American Journal of Public Health, vol. 95, no. 5, pp. 851-859, 2005.
[18] U.S. Department of Justice, Office of Justice Programs, and Bureau of Justice Statistics, A Bureau of Justice Statistical Profile, 1992-2002: AI/ANs and Crime, 2004, http://www.bjs .gov/content/pub/pdf/aic02.pdf.

[19] D. J. English, D. B. Marshall, S. Brummel, and M. Orme, "Characteristics of repeated referrals to child protective services in Washington state," Child Maltreatment, vol. 4, no. 4, pp. 297307, 1999.

[20] R. F. Anda, D. W. Brown, V. J. Felitti, S. R. Dube, and W. H. Giles, "Adverse childhood experiences and prescription drug use in a cohort study of adult HMO patients," BMC Public Health, vol. 8, article 198, 2008.

[21] Indian Health Service, Indian Health Service National Tribal Advisory Committee on Behavioral Health, and Indian Health Service Behavioral Health Work Group, American Indian/Alaska Native National Behavioral Health Strategic Plan 2011-2015, 2011, http://www.nihb.org/docs/08072012/ AIANNationalBHStrategicPlan.pdf.

[22] D. S. Bigfoot and S. R. Schmidt, "Honoring children, mending the circle: cultural adaptation of trauma-focused cognitivebehavioral therapy for american indian and alaska native children," Journal of Clinical Psychology, vol. 66, no. 8, pp. 847856, 2010.

[23] M. Chaffin, D. Bard, D. S. BigFoot, and E. J. Maher, "Is a structured, manualized, evidence-based treatment protocol culturally competent and equivalently effective among American Indian parents in child welfare?" Child Maltreatment, vol. 17, no. 3, pp. 242-252, 2012.

[24] A. Barlow, B. Mullany, N. Neault et al., "Paraprofessionaldelivered home-visiting intervention for American Indian teen mothers and children: 3-year outcomes from a randomized controlled trial," American Journal of Psychiatry, vol. 172, no. 2, pp. 154-162, 2015.

[25] Patient Protection and Affordable Care Act. Pub. L. No. 111148, 124 Stat. 334, March 2010, https://www.congress.gov/111/ plaws/publ148/PLAW-111publ148.pdf. 


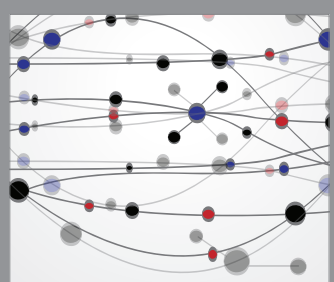

The Scientific World Journal
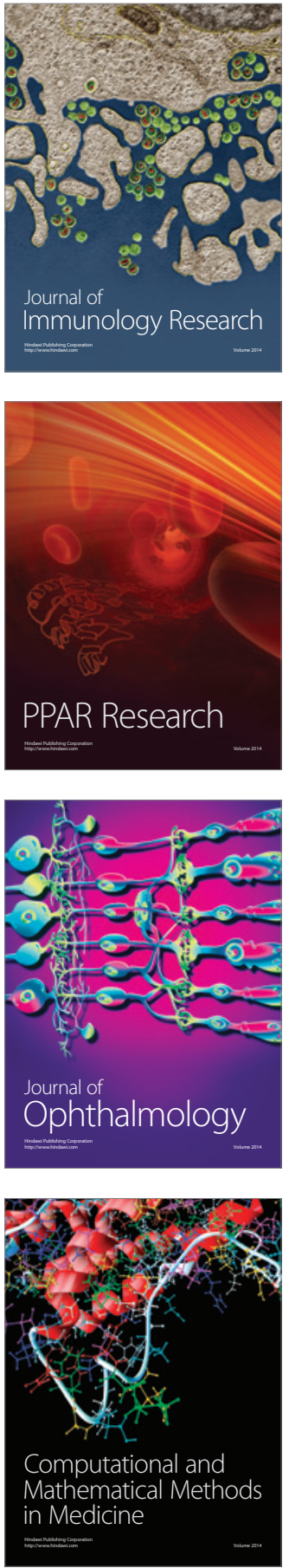

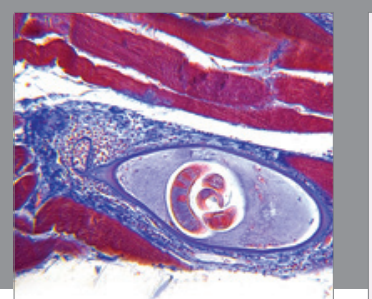

Gastroenterology Research and Practice

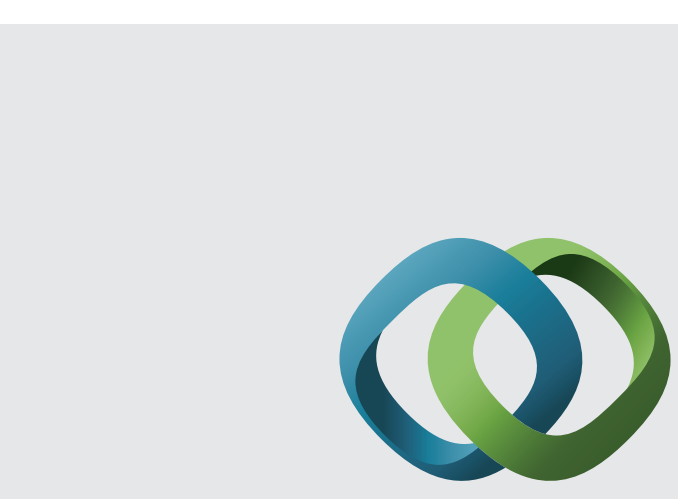

\section{Hindawi}

Submit your manuscripts at

http://www.hindawi.com
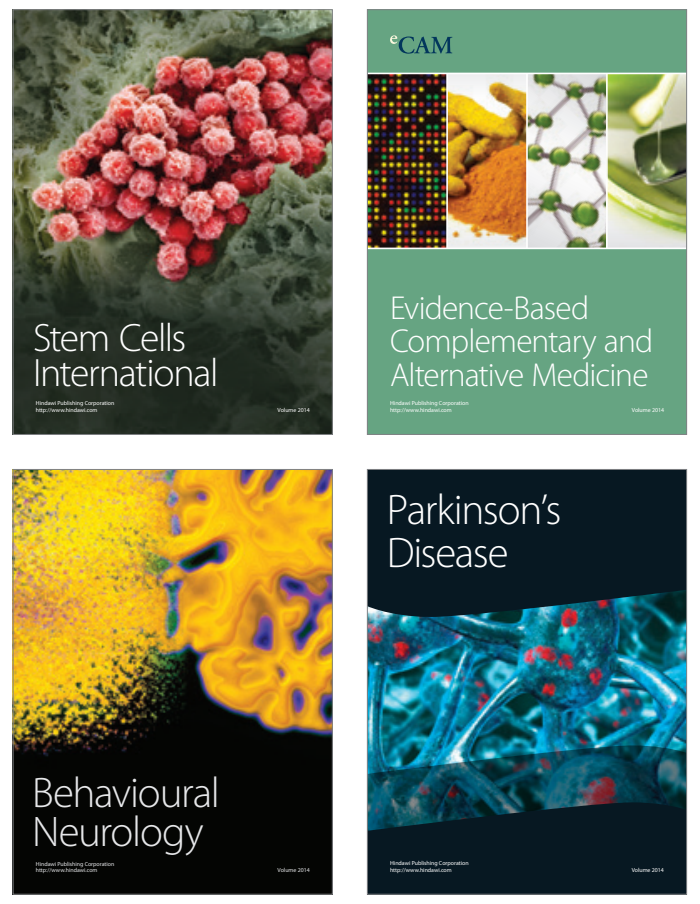
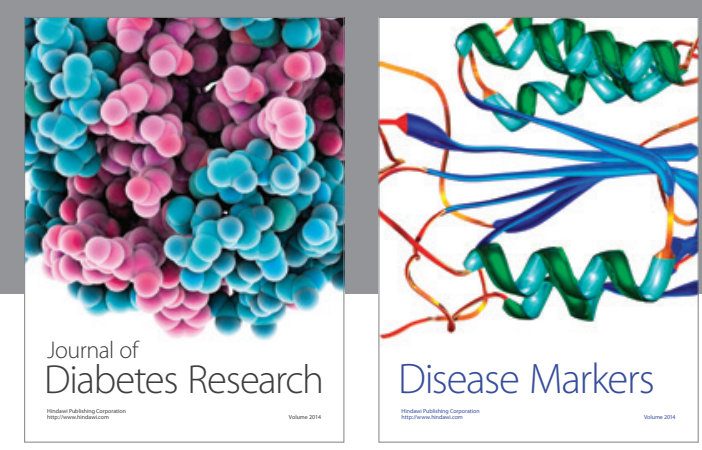

Disease Markers
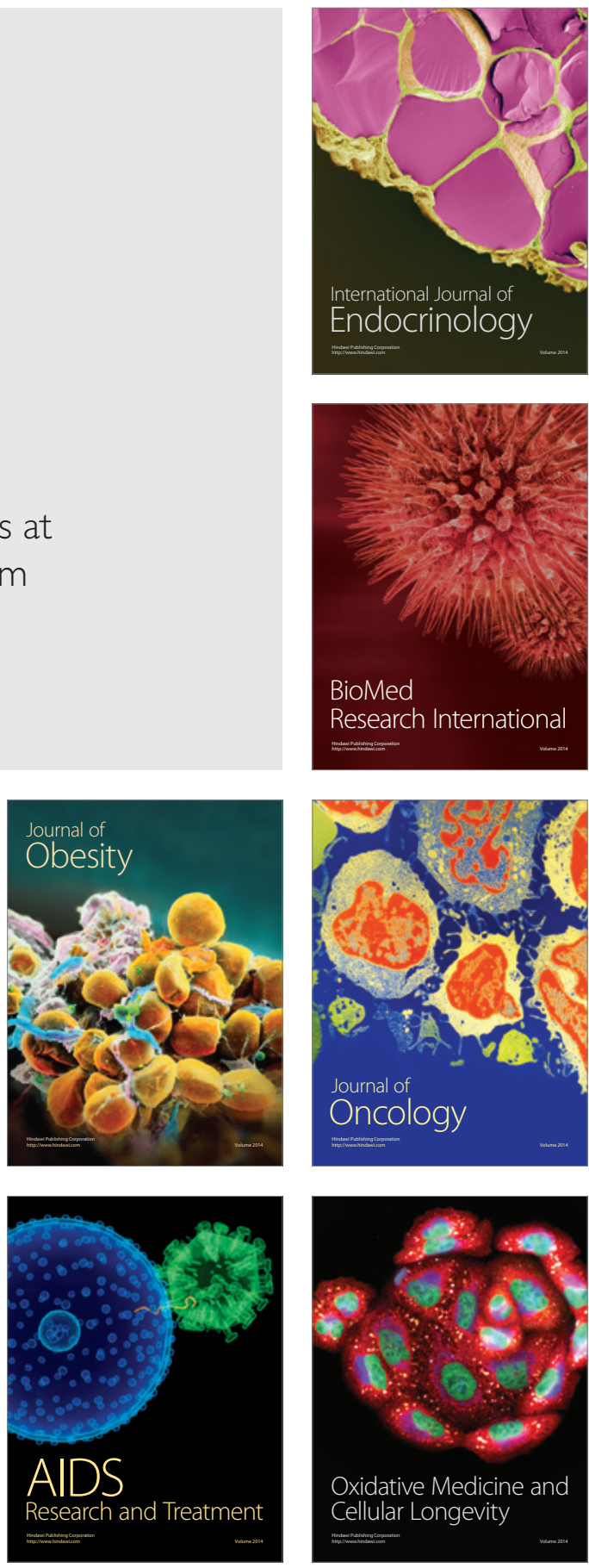